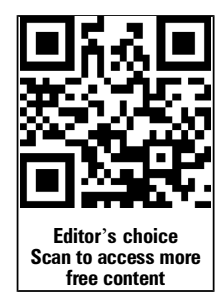
Hospital, HOB, Brasilia, DF, Brazil

${ }^{2}$ Ophthalmology Department Faculdade de Medicina, Universidade de São Paulo, São Paulo, SP, Brazil

\section{Correspondence to}

Dr Patrick F Tzelikis, Brasilia Ophthalmologic Hospital, HOB SQN 203, bloco K, apart 502 Brasilia, DF 70833-110, Brazil; tzelikis@gmail.com

Received 15 August 2014 Revised 16 October 2014 Accepted 22 October 2014 Published Online First 10 November 2014

\title{
Comparison of ketorolac $0.4 \%$ and nepafenac $0.1 \%$ for the prevention of cystoid macular oedema after phacoemulsification: prospective placebo-controlled randomised study
}

\author{
Patrick Frensel Tzelikis, ${ }^{1}$ Monike Vieira, ${ }^{1}$ Wilson Takashi Hida ${ }^{1}, 2$ \\ Antonio Francisco Motta, ${ }^{2}$ Celso Takashi Nakano, ${ }^{2}$ Eliane Mayumi Nakano, ${ }^{1}$ \\ Milton Ruiz Alves ${ }^{2}$
}

\begin{abstract}
Purpose To compare the anti-inflammatory efficacy of ketorolac of tromethamine $0.4 \%$ and nepafenac $0.1 \%$ eye drops for prophylaxis of cystoid macular oedema (CME) after small-incision cataract extraction. Methods Patients were assigned randomly to three groups. Group 1 patients received a topical artificial tear substitute (placebo); group 2 received ketorolac tromethamine $0.4 \%$ (Acular LS, Allergan) and group 3 received nepafenac $0.1 \%$ (Nevanac, Alcon). The incidence and severity of CME were evaluated by retinal foveal thickness on optical coherence tomography (OCT) after 1, 4 and 12 weeks.

Results One hundred and twenty-six eyes of 126 patients were included in this study. The between-group differences in visual outcomes, central corneal thickness and endothelial cell density were not statistically significant. In all retinal thickness measurements, an increase was detected starting from the postoperative first week until 12 weeks. There was no statistically significant difference between the three groups in any measurement performed by spectral-domain OCT.

Conclusions Used prophylactically after uneventful cataract surgery, non-steroidal anti-inflammatory drugs were not efficacious in preventing macular oedema compared with placebo.

Trial registration number ClinicalTrials: NCT02084576.
\end{abstract}

\section{INTRODUCTION}

Advances in intraocular lens (IOL), phacoemulsification technology and improved incision designs have resulted in decreased intraocular inflammation following cataract extraction. Despite improvements in technique, many patients still exhibit clinically significant inflammation after cataract surgery that requires treatment with an inflammatory eyedrop. ${ }^{1}$ Left untreated, inflammation can lead to many complications including pain and photophobia, elevated intraocular pressure (IOP), posterior synechiae and cystoid macular oedema (CME). Topical non-steroidal anti-inflammatory drugs (NSAIDs) diminish inflammation and discomfort after surgery. ${ }^{2}$ The advantages of NSAIDs over corticosteroids include stability of IOP, reduced risk of secondary infections and benefit of some analgesia. $^{3-5}$ To prevent inflammation after cataract surgery, steroids and NSAIDs are agents at our disposal, but there is still debate over whether to use them in combination for routine cases.

Despite a convincing theory of pathogenesis involving migratory inflammatory mediator and hyperpermeability of retinal blood vessels, particularly the retinal capillary bed, evidence-based medicine still regards prophylaxis and treatment with anti-inflammatory agents as unproven. The present study evaluated the effects of ophthalmic solutions of the NSAIDs ketorolac $0.4 \%$ and nepafenac $0.1 \%$ in preventing CME assessed by optical coherence tomography (OCT) using central subfield thickness $(\mathrm{CST}, \mu \mathrm{m})$ and total macular volume (TMV, $\mathrm{mm}^{3}$ ) as the indicator of macular swelling 1, 4 and 12 weeks after phacoemulsification cataract extraction.

\section{METHODS \\ Patient enrolment}

This prospective randomised clinical trial study included patients older than 40 years with age-related cataract, and a normal ophthalmological examination besides senile cataract. Written informed consent was obtained from all patients before surgery and the local ethics committee approved the study. All the procedures were in accordance with the ethical standards of the responsible committee on human experimentation and with the Helsinki Declaration of 1975, which was revised in 1983. Exclusion criteria were previous ocular surgery, central endothelial cell count $<2000$ cells $/ \mathrm{mm}^{2}$, glaucoma or IOP $>21 \mathrm{~mm} \mathrm{Hg}$, amblyopia, retinal abnormalities, steroid or immunosuppressive treatment, connective tissue diseases or an allergy or hypersensitivity to NSAIDs. Enrolled patients who had complicated cataract surgery (eg, posterior capsule rupture, vitreous loss or an IOL not placed in the capsular bag) were subsequently excluded.

\section{Study protocol}

Preoperatively, patients had an extensive ophthalmological examination, including measurement of corrected distance visual acuity (CDVA), refraction, slit-lamp examination, IOP, ophthalmoscopy, corneal endothelial cell count by non-contact specular microscopy (Noncon Robo, Konan), central corneal thickness (CCT) measurement (Pentacam, Oculus) and biometry with the IOLMaster partial coherence interferometry device (Carl Zeiss Meditec AG). The targeted postoperative refractive 
error was $0.0 \mathrm{D}$. The visual acuity measurements were recorded with $\log$ MAR uncorrected distance visual acuity and CDVA.

In addition, a baseline spectral-domain OCT (SD-OCT) scan was performed before surgery and postoperatively after 1, 4 and 12 weeks. All SD-OCT imaging was performed with a spectraldomain Heidelberg Spectralis OCT devise (Heidelberg Engineering, Heidelberg, Germany). The following scan acquisition parameters were required: dense volume scan $\left(20^{\circ} \times 20^{\circ}\right.$, roughly $6 \times 6 \mathrm{~mm}), 49 \mathrm{~B}$-scans each spaced $120 \mu \mathrm{m}$ apart, automatic real-time mean of 16 , high speed (512 A-scans/B-scans) and software V.5.3 ${ }^{\mathrm{a}}$. V.5. $33^{\mathrm{a}}$ calculates retinal volume over the entire $6 \mathrm{~mm}$ Early Treatment Diabetic Retinopathy Study grid (as long as at least 50\% of individual subfields are scanned). Specifically, CST $(\mu \mathrm{m})$ and TMV $\left(\mathrm{mm}^{3}\right)$ were collected from each patient for statistical analysis. The SD-OCT uses the posterior border of Bruch's membrane as the boundary for retinal thickness measurements.

Patients were assigned in a 1:1:1 ration to one of three treatment groups using a computer-generated randomisation list. The study medications were artificial tear substitute (ATS) in group 1; ketorolac tromethamine $0.4 \%$ (Acular LS, Allergan) in group 2 and nepafenac $0.1 \%$ (Nevanac, Alcon) in group 3. Patients were instructed to instil one drop of ATS in the operative eye four times a day (breakfast, lunch, dinner and before bedtime), one drop of ketorolac tromethamine $0.4 \%$ four times a day and one drop of nepafenac $0.1 \%$ three times a day. They began dosing 2 days before surgery and continued for 4 weeks. In addition, patients received moxifloxacin $0.5 \%$ drops four times a day starting 2 days before surgery and continued for 7 days after surgery. All patients received on day 0 prednisolone $1 \%$ drops four times a day for 1 week, three times a day for 1 week, two times a day for 1 week and one time a day for 1 week. All investigators were masked with regard to treatment group.

\section{Surgical technique}

All operations were performed in a standard way by the same experienced surgeon. Briefly, phacoemulsification surgery was carried out via a temporal clear corneal incision, and a foldable posterior chamber IOL was implanted into the capsular bag (Acrysof SN60WF, Alcon Surgical). The same irrigation solution (BSS solution, Alcon, Fort Worth, Texas, USA) and ophthalmic viscoelastic device (sodium hyaluronate 3.0\%-chondroitin sulfate 4.0\%, Viscoat; Alcon, Fort Worth, Texas, USA) were used in all cases. The surgeon was prohibited from using intracameral lidocaine or antibiotics.

\section{Statistical analysis}

Data were entered into a Microsoft Excel spreadsheet and analysed using SPSS (V.17.0, SPSS). Quantitative variables are described using mean $\pm S D$ or median as well as minimum and maximum value where appropriate. To test whether the three medications had comparable baseline values concerning the target variables, a one-way repeated measures analysis of variance (ANOVA) or Kruskal-Wallis test were applied depending on sample distribution. The one-way repeated measures ANOVA was used to check quantitative variables for changes over time. Any differences showing a $\mathrm{p}$ value of $<0.05$ (ie, at the $5 \%$ level) were considered to be statistically significant.

\section{RESULTS}

\section{Patient characteristics}

Patients were recruited for this study from only one site between 4 June 2013 and 7 October 2013. A total of 150 patients were screened and consented to participate in this study. Ten patients decided not to participate, eight were lost to follow-up and eight were discontinued because of protocol violations. A total of 126 patients (40 in the ATS group, 45 in the ketorolac group and 41 in the nepafenac group) underwent surgery and entered the trial. There were no adverse side effects in either group.

Fifty-six were men (44.0\%) and 70 were women $(56.0 \%)$. The mean age of the patients was 65.36 years, range from 50 to 90 years (SD \pm 7.3$)$, they all had a mild cataract at presentation. The mean spherical power of the implanted IOLs was +21.67 $\pm 3.1 \mathrm{D}$ (range 12.00-30.00 D). The mean preoperative axial length (AL) was $23.58 \pm 0.8 \mathrm{~mm}$ (range $21.00-27.30 \mathrm{~mm}$ ). Patients were followed up for 12 weeks. There were no differences in age, sex, IOL power and AL ( $p>0.05)$.

\section{Visual acuity and refraction}

Table 1 shows the CDVA. Postoperatively, the CDVA was 20/40 or better in all eyes (126 eyes; 100\%), 20/25 or better in 102 eyes $(81.0 \%)$ and $20 / 20$ or better in 93 eyes $(73.8 \%)$. At 4 weeks, the CDVA improved in all groups; however, there was no statistically significant difference in mean values between the placebo group $(0.02 \pm 0.05 \log \mathrm{MAR})$, the ketorolac group $(0.06$ $\pm 0.12 \log$ MAR $)$ or the nepafenac group $(0.04 \pm 0.10 \log \mathrm{MAR})$.

\section{Central corneal thickness and endothelial cell density}

Table 1 summarises the results of each group. The difference in CCT and endothelial cell density (ECD) was not statistically significant between the three groups ( $p>0.05$, ANOVA).

\section{Optical coherence tomography}

Tables 2 and 3 show the preoperative and postoperative thickness values of the retinal layers in the control group, ketorolac group and nepafenac group. There was no statistically significant difference between the three groups in any measurement performed by SD-OCT.

All three groups had varying statistically significant intraocular differences between baseline and 1,4 and 12 weeks. In all retinal thickness measurements, an increase was detected

Table 1 Preoperative and 30 days postoperative data

\begin{tabular}{|c|c|c|c|c|c|c|c|c|}
\hline & \multicolumn{2}{|l|}{$\begin{array}{l}\text { Group } 1 \\
\text { Mean } \pm \text { SD }\end{array}$} & \multicolumn{2}{|l|}{$\begin{array}{l}\text { Group } 2 \\
\text { Mean } \pm \text { SD }\end{array}$} & \multicolumn{2}{|l|}{$\begin{array}{l}\text { Group } 3 \\
\text { Mean } \pm \text { SD }\end{array}$} & \multicolumn{2}{|l|}{ p Value } \\
\hline & Preop & Postop & Preop & Postop & Preop & Postop & Preop & Postop \\
\hline CDVA (logMAR) & $0.13(0.17)$ & $0.01(0.05)$ & $0.16(0.13)$ & $0.06(0.12)$ & $0.17(0.20)$ & $0.04(0.10)$ & 0.74 & 0.44 \\
\hline $\mathrm{CCT}(\mu \mathrm{m})$ & $529.93(32.37)$ & $527.10(32.93)$ & $517.78(40.84)$ & 518.09 (39.07) & $535.81(39.85)$ & 531.76 (38.89) & 0.15 & 0.33 \\
\hline $\mathrm{ECD}$ (cells $/ \mathrm{mm}^{2}$ ) & 2495.35 (323.69) & $2323.23(320.70)$ & $2288.11(415.11)$ & $2164.90(493.86)$ & $2340.75(425.64)$ & $2168.75(539.17)$ & 0.24 & 0.48 \\
\hline
\end{tabular}

CCT, central corneal thickness; CDVA, corrected distance visual acuity; ECD, endothelial cell density. 
Table 2 Between-group comparison of central foveal thickness (CFT, $\mu \mathrm{m})$ measured with SD-OCT

\begin{tabular}{lllll}
\hline \multicolumn{5}{c}{ Postoperative } \\
\cline { 3 - 5 } Group & Baseline & 1 week & 4 weeks & 12 weeks \\
\hline Group 1: control & & & \\
Mean \pm SD & $259.93(30.84)$ & $268.86(30.51)$ & $270.71(30.73)$ & $274.82(30.45)$ \\
Group 2: ketorolac & & & \\
Mean \pm SD & $269.78(34.69)$ & $271.46(37.42)$ & $278.85(45.43)$ & $282.26(45.21)$ \\
Group 3: nepafenac & & & \\
Mean $\pm S D$ & $270.44(33.68)$ & $274.01(33.48)$ & $279.00(33.82)$ & $289.73(54.80)$ \\
p Value & 0.56 & 0.89 & 0.77 & 0.54 \\
\hline SD-OCT, spectral-domain optical coherence tomography.
\end{tabular}

starting from the postoperative first week until 12 weeks. The statistically significant increases in postoperative retinal thickness measurements were maintained until 12-week control visits in all groups studied.

Figure 1 shows the percentage of patients in whom the postoperative check-up revealed a change in central subfields retinal thickness of $\leq 10 \mu \mathrm{m}$, between 10 and $25 \mu \mathrm{m}$, and $>25 \mu \mathrm{m}$ compared with preoperative values in all groups. The highest percentages of $>10 \mu \mathrm{m}$ were observed in the group 1. In this case, as well, the ANOVA test was not significant between the groups.

Figures 2 and 3 show the percentage of patients in whom we defined macular oedema using an upper cut-off of two and three SDs above the mean baseline CST by treatment group.

\section{DISCUSSION}

CME remains a common cause of vision loss after cataract surgery. ${ }^{6}$ Fluorescein angiography has been the traditional method used to confirm its presence with retinal telangiectasis in the early phase frames and accumulation of fluorescein in a petaloid pattern surrounding the fovea in late phase frames. OCT has emerged as an important imaging modality in the evaluation and management of retinal diseases. Since the late 1990 s, the interpretation and management of macular pathologies have undergone a major change with the ability of OCT to non-invasively image intraocular structures in vivo. ${ }^{7}$ Macular thickness, as measured by OCT, is one of the key outcome measures used in most clinical trials, especially those on diabetic macular oedema and age-related macular degeneration and has been increasingly used to assess CME after cataract surgery. ${ }^{7} 8$

Our results show that 1,4 and 12 weeks after uneventful phacoemulsification cataract extraction, there was no difference in

Table 3 Between-group comparison of total macular volume $\left(\mathrm{TMV}, \mathrm{mm}^{3}\right.$ ) measured with SD-OCT

\begin{tabular}{|c|c|c|c|c|}
\hline \multirow[b]{2}{*}{ Group } & \multirow[b]{2}{*}{ Baseline } & \multicolumn{3}{|c|}{ Postoperative } \\
\hline & & 1 week & 4 weeks & 12 weeks \\
\hline \multicolumn{5}{|c|}{ Group 1: control } \\
\hline Mean \pm SD & $8.56(0.64)$ & $8.57(0.56)$ & $8.69(0.58)$ & $8.72(0.61)$ \\
\hline \multicolumn{5}{|c|}{ Group 2: ketorolac } \\
\hline Mean \pm SD & 7.99 (1.31) & $8.17(1.20)$ & $8.33(1.23)$ & $8.38(1.25)$ \\
\hline \multicolumn{5}{|c|}{ Group 3: nepafenac } \\
\hline Mean \pm SD & 7.89 (1.32) & $8.16(1.23)$ & $8.21(1.23)$ & $8.21(1.23)$ \\
\hline $\mathrm{p}$ Value & 0.20 & 0.47 & 0.43 & 0.37 \\
\hline
\end{tabular}

SD-OCT, spectral-domain optical coherence tomography.

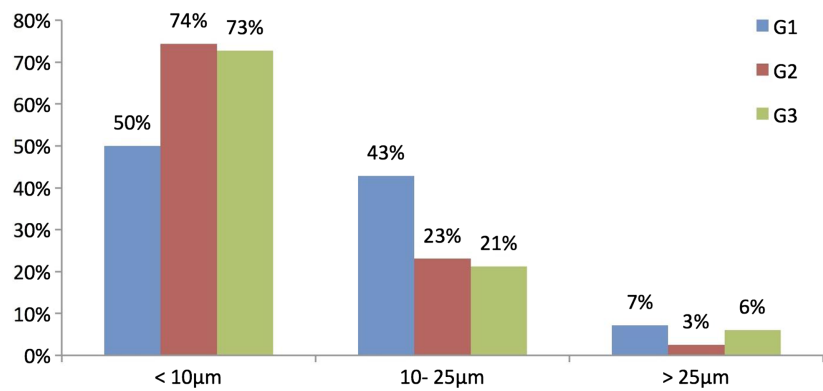

Figure 1 Percentage of eyes with a change in central subfield retinal thickness at 4 weeks compared with the preoperative examination by treatment group (G1=placebo; G2=ketorolac and G3=nepafenac).

CST $(\mu \mathrm{m})$ and TMV $\left(\mathrm{mm}^{3}\right)$ between a placebo, ketorolac and nepafenac. In a similar study by Almeida et $a l^{9}$ evaluating the efficacy of prophylactic NSAIDs versus placebo on macular volume 1 month after phacoemulsification, in patients with no risk factors for macular oedema and no intraoperative complications, the authors found no statistically significant differences between the three study groups $(p=0.29)$. This study concluded that in low-risk patients, prophylactic use of topical ketorolac or nepafenac seems to offer no benefit in preventing OCT changes indicative of macular oedema after surgery. However, in another previous study by the same authors, Almeida et al, ${ }^{10}$ comparing ketorolac versus placebo in at-risk patients with uveitis, diabetes or macular disease, found that ketorolac significantly decreased macular volume after cataract surgery. The recommendation remains that for at-risk patients, prophylactic use of topical NSAIDs is an efficacious and safe intervention to minimise postoperative macular oedema.

Our study also shows an increase in macular thickness detected by SD-OCT after surgery, starting from the postoperative first week and remaining for at least 12 weeks in all groups studied. It is usually thought that retinal thickness would be increased after cataract surgery due to postoperative inflammation, especially without using NSAIDs. Biro et al, ${ }^{11}$ in a study that measured the foveal and perifoveal thickness of the retina after phacoemulsification found a significant increase oedema detected using OCT at postoperative day 7 to 6 months. The initial preoperative average value of $234.1 \pm 2.6 \mu \mathrm{m}$ in the $6.0 \mathrm{~mm}$ perifoveal region increased to $242.5 \pm 2.6 \mu \mathrm{m} 1$ week, to $247.7 \pm 4.6 \mu \mathrm{m} 1$ month and to $246.0 \pm 5.9 \mu \mathrm{m} 2$ months after surgery. Binder ${ }^{12}$ also conducted a similar study and reported the presence of macular oedema especially prominent in the perifoveal regions, starting from the postoperative seventh day, which continued postoperatively until 6 months. This is consistent with our finding that the majority of eyes after cataract surgery demonstrate at least minimal increases in OCT thickness.

Donnenfeld $e \mathrm{al}^{13}$ more formally assessed the hypothesis that NSAIDs may be able to reduce the incidence of CME as well as control postoperative inflammation. This randomised, singlepractice, blinded trial of 100 patients evaluated various preoperative dosing regiments of ketorolac versus placebo. This study did not report such definitive results regarding the reduction of CME. At 2 weeks, no patients in the ketorolac 3-day or 1 -day pretreatment groups $(n=25$ each) had clinically significant CME, while three out of 25 patients in the control group did. There are two considerations to be examined. First, the reported between-group difference was not significant $(p=0.023)$, just like our study after 4 weeks $(p=0.77)$, although this may be secondary to the small sample size. Next, the peak prevalence of acute CME appears about 4-6 weeks after cataract surgery, ${ }^{11} 14$ 


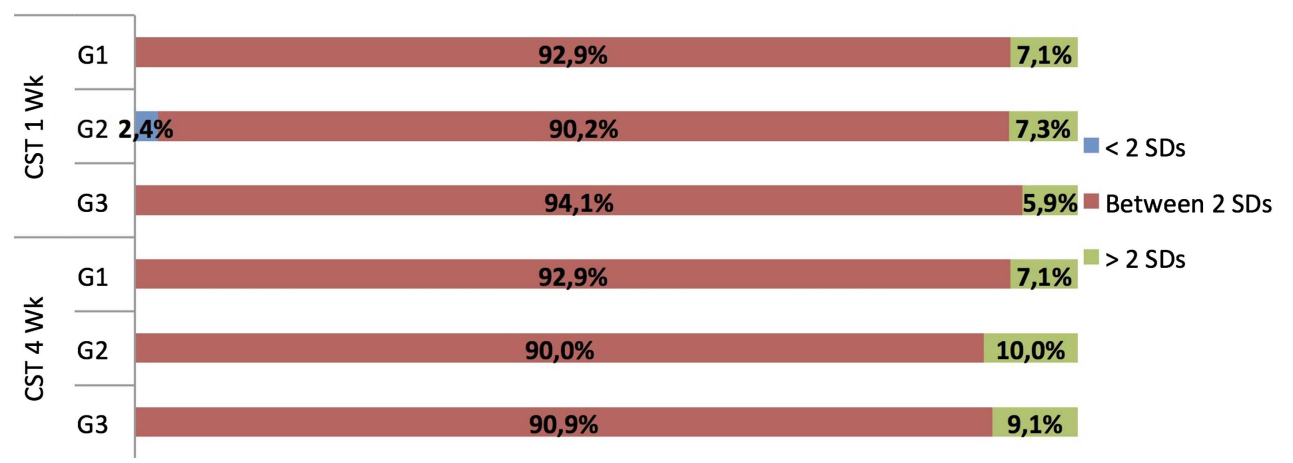

Figure 2 Percentage of patients in whom we defined macular oedema using an upper cut-off of two SDs above the mean baseline central subfield thickness (CST) by treatment group (G1=placebo; G2=ketorolac and G3=nepafenac).

Figure 3 Percentage of patients in whom we defined macular oedema using an upper cut-off of three SDs above the mean baseline central subfield thickness (CST) by treatment group (G1=placebo, G2=ketorolac, and $\mathrm{G} 3=$ nepafenac).

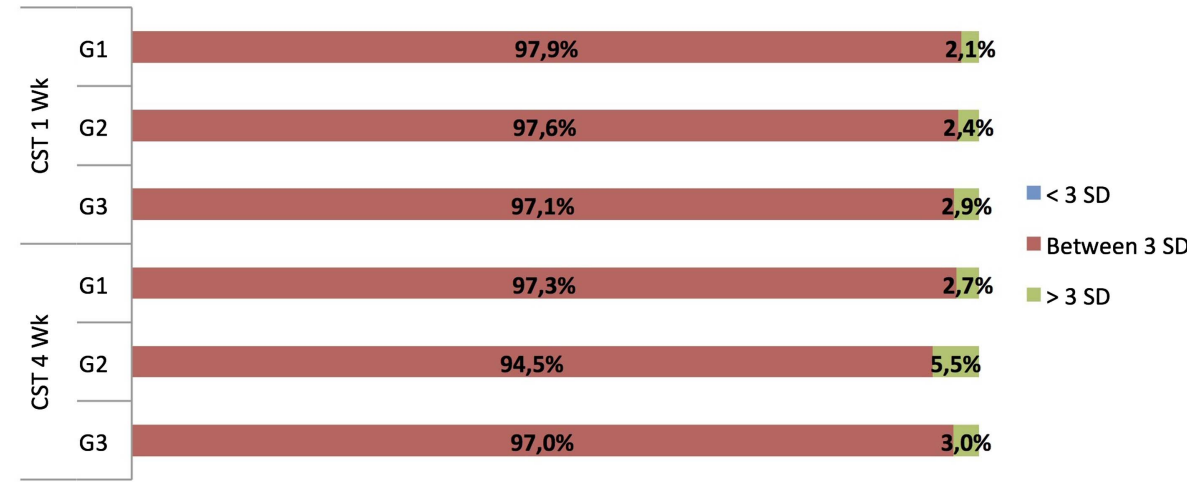

whereas the study of Donnenfeld et al only monitored CME for 2 weeks, different from our study that followed up the patients until 12 weeks.

There is no consensus on OCT parameters for identifying the presence of CME after cataract surgery. A prospective study by Perente et al ${ }^{15}$ involving 110 eyes of 102 healthy patients reported a peak incidence rate of CME, as defined it, of $10.9 \%$ at 4 weeks using OCT after uneventful cataract surgery. All eyes had preoperative OCTs performed with a mean CST (central $1.0 \mathrm{~mm}$ diameter) of $202.4 \pm 25.9 \mu \mathrm{m}$ and were evaluated postoperatively at regular intervals with OCT. The authors defined CME as two SDs above the preoperative mean CST $(\geq 225 \mu \mathrm{m})$. Using this definition, the authors also observed a $3.6 \%$ incidence rate of CME at 180 days. The study was unable to show a strong correlation between eyes with CME and vision loss, which further suggests that their incidence rate may have been overestimated. Kim et al $^{16}$ showed that a $40 \%$ increase in baseline centre point thickness accurately determined $100 \%$ of eyes with macular oedema and $99 \%$ of eyes without macular oedema and detected that the incidence of postoperative macular oedema was $14 \%$ at 1 month for diabetic, uveitic and healthy eyes. Kusbeci et $a l^{17}$ defined CME as three SDs above the preoperative mean central macular thickness. Using this definition, they observed a $5.5 \%$ and $4.4 \%$ incidence rate of $\mathrm{CME}$ at postoperative 12 weeks and 24 weeks, respectively. Using the definition of two SDs, we observed a higher incidence rate of 7.1\%, 10.0\% and 9.1\% of $\mathrm{CME}$ at postoperative 4 weeks in placebo, ketorolac and nepafenac, respectively. When we used three SDs we could observe a reduction in the incidence rate to $2.1 \%, 2.4 \%$ and $2.9 \%$ of $\mathrm{CME}$ also at postoperative 4 weeks in the placebo, ketorolac and nepafenac groups, respectively.
In the current study, visual outcomes were comparable and not statistically significant between groups. The findings in our study also demonstrate that NSAIDs had no efficacy in improving measurable surgical outcomes like CCT and endothelial cell loss. These findings are worse than those of Donnenfeld et al ${ }^{13}$ who report that 3-day and 1-day dosing of ketorolac reduced surgical time, endothelial cell loss and improved visual acuity in the immediate postoperative period compared with placebo. In another study that evaluated the efficacy of prophylactic ketorolac versus nepafenac versus placebo by Almeida et $a l^{9}$ there was no difference in mean values or ranked mean values of CDVA between the placebo group and the NSAIDs groups after 1 month of follow-up.

In conclusion, visual recovery, CCT, ECD, macular foveal thickness and TMV were statistically similar between the placebo group, ketorolac group and the nepafenac group. Establishing normal incidence ranges of CME using OCT and clarifying its correlation with vision will become increasingly important. There are several limitations to our study including our placebo group that was likely too small to detect all associated conditions we want. Future studies with larger sample sizes are necessary to better define these associations.

Contributors By submitting this manuscript, each of the authors indicates that they had full access to all of the data in this study and take complete responsibility for the integrity of the data and the accuracy of the data analysis. Conception and design: PFT, MV, WTH, AFM, CTN, EMN and MRA. Analysis and interpretation: PFT, WTH, AFM, CTN and EMN. Writing the article: PFT, MV, WTH, AFM, CTN, EMN and MRA. Data collection: MV and AFM. Statistical expertise: PFT. Final approval of the article: PFT, MV, WTH, AFM, CTN, EMN and MRA.

Competing interests None.

Patient consent Obtained. 
Ethics approval Local Ethics Committee.

Provenance and peer review Not commissioned; externally peer reviewed.

\section{REFERENCES}

1 Flach AJ, Lavelle CJ, Olander KW, et al. The effect of ketorolac tromethamine solution $0.5 \%$ in reducing postoperative inflammation after cataract extraction and intraocular lens implantation. Ophthalmology 1988:95:1279-84.

2 Flach AJ, Dolan BJ, Donahue ME, et al. Comparative effects of ketorolac $0.5 \%$ or diclofenac $0.1 \%$ ophthalmic solutions on inflammation after cataract surgery. Ophthalmology 1998;105:1775-9.

3 Roberts CW, Brennan KM. A comparison of topical diclofenac with prednisone for postcataract inflammation. Arch Ophthalmol 1995;113:725-7.

4 Rossetti L, Bujtar E, Castoldi D, et al. Effectiveness of diclofenac eyedrops in reducing inflammation and the incidence of cystoid macular edema after cataract surgery. J Cataract Refract Surg 1996;22:794-9.

5 Wright PL, Wilkinson CP, Balyeat HD, et al. Angiographic cystoid macular edema after posterior chamber lens implantation. Arch Ophthalmol 1988;106:740-4.

6 Nelson ML, Martinin A. managing cystoid macular edema after cataract surgery. Curr Opin Ophthalmol 2003;14:39-43.

7 Puliafito CA, Hee MR, Lin CP, et al. Imaging of macular diseases with optical coherence tomography. Ophthalmology 1995;102:217-29.

8 Srinivasan VJ, Wojtkowski M, Witkin AJ, et al. High-definition and 3-dimentional imaging of macular pathologies with high-speed ultrahigh-resolution optical coherence tomography. Ophthalmology 2006;113:2054-65.
9 Almeida DRP, Khan Z, Xing L, et al. Prophylactic nepafenac and ketorolac versus placebo in preventing postoperative macular edema after uneventful phacoemulsification. J Cataract Refract Surg 2012;38:1537-43.

10 Almeida DRP, Johnson D, Hollands $H$, et al. Effect of prophylactic nonsteroidal anti-inflammatory drugs on cystoid macular edema assessed using optical coherence tomography quantification of total macular volume after cataract surgery. J Cataract Refract Surg 2008;34:64-9.

11 Biro Z, Balla Z, Kovacs B. Change of foveal and perifoveal thickness measured by OCT after phacoemulsification and IOL implantation. Eye 2008;22:8-12.

12 Binder SP. OCT detects higher than expected incidence of macular oedema after cataract surgery. Eurotimes 2004;9:1-3.

13 Donnenfeld ED, Perry HD, Wittpenn JR, et al. Preoperative ketorolac tromethamine $0.4 \%$ in phacoemulsification outcomes: pharmacokinetic-response curve. J Cataract Refract Surg 2006;32:1474-8.

14 Kim SJ, Belair ML, Bressler NM, et al. A method of reporting macular edema after cataract surgery using optical coherence tomography. Retina 2008;28:870-6.

15 Perente I, Utine CA, Cakir $\mathrm{H}$, et al. Evaluation of macular changes after uncomplicated phacoemulsification surgery by optical coherence tomography. Curr Eye Res 2007;32:241-7.

16 Kim SJ, Equi R, Bressler NM. Analysis of macular edema after cataract surgery in patients with diabetes using optical coherence tomography. Ophthalmology 2007;114:881-9.

17 Kusbeci T, Eryigit L, Yavas G, et al. Evaluation of cystoid macular edema using optical coherence tomography and fundus fluorescein angiography after uncomplicated phacoemulsification surgery. Curr Eye Res 2012;37:327-33. 